\title{
Application of Löwdin Projectors to Evaluate Density Matrix Evolutions
}

\author{
N. CHANDRAKUMAR \\ Laboratory of Chemical Physics, Central Leather Research Institute, \\ Adayaru, Madras 600020 . Tamil Nadu, India
}

Received November 9, 1989

\begin{abstract}
Projection operators are employed to evaluate functions of operators. In particular, this formalism is used to find polynomial expressions for exponential operators and thence to solve density matrix evolutions in closed analytical form. Problems explicitly treated include pulse rotation of spin- 1 and spin- $\frac{3}{2}$ systems, spin tickling and spin interferometry on a system of two coupled spin- $\frac{1}{2}$ nuclei, isotropic mixing in systems of two coupled nuclei (both spins $-\frac{1}{2}$ or spins-1), and dipolar evolution in a two-spin- $-\frac{1}{2}$ system. The method is compared to the $\mathrm{BCH}$ approach for operator evolutions. 1990 Academic Press, Inc.
\end{abstract}

Time evolution of a physical system is governed in quantum mechanics by the Schrödinger equation, or equivalently the Liouville-von Neumann equation for ensembles. Formally, the time evolution may be effected by a time displacement operator. The Hamiltonian $\mathscr{H}$ of the system is the generator of infinitesimal time translations; in formal descriptions of evolution over finite time durations, the Hamiltonian therefore gets exponentiated and acts on the initial state of the system. Thus, with a time-independent Hamiltonian, the evolution of the density matrix $\rho$ for an ensemble may be written as

$$
\rho(t)=\exp (-i \mathscr{H} t) \rho(0) \exp (i \mathscr{H} t) .
$$

In the average-Hamiltonian approach, the Magnus expansion or Floquet theory (14 ) is employed to cast evolutions under a time-dependent Hamiltonian in the same form, with the leading term $\overrightarrow{\mathscr{H}}$ of these expansions substituted, to zeroth order, in place of $\mathscr{H}$ in [1]. In either case, the evaluation of the right-hand side of [1] leads to the desired expression for the density operator as a function of time. Numerical or analytical solution of the individual elements of $\rho$ is a popular approach; however, there is considerable interest in obtaining a closed form analytical solution to the problem, employing especially one of the several operator representations of $\rho$ and the appropriate commutator algebra (5-7). In this work we point out and illustrate a complementary approach, obtaining explicit polynomial expressions for the exponential operators and proceeding then to analytically evaluate the right-hand side of [1].

\section{THEORY}

In the basic theory of normal operators, well-known methods are available to evaluate operator functions in terms of the spectrum of the operator. These methods bear 
a close relationship to the Cayley or Cayley-Hamilton theorem of matrix algebra $(8,9)$. For our purposes, however, the geometrically appealing methods of spectral decomposition and projection operators introduced by Löwdin appear particularly attractive (10). The resulting expression for a function $f(A)$ of an operator $A$ is given as

$$
f(A)=\sum_{i} f\left(\lambda_{i}\right) P_{\lambda_{i}}
$$

the $\lambda_{i}$ values being the eigenvalues of $A$; the projectors $P_{\lambda_{i}}$ are given by

$$
P_{\lambda_{i}}=\prod_{j \neq i} \frac{\left(A-\lambda_{j}\right)}{\left(\lambda_{i}-\lambda_{j}\right)} .
$$

The sum in [2] -as also the product in [3] -runs over all distinct eigenvalues of $A$, each degenerate eigenvalue being accounted exactly once. The equivalence of [3] to the Sylvester matrix of $A$ may also be noted, again bringing into correspondence the geometric and algebraic viewpoints. Note that the operators $P$ are Hermitian, idempotent operators. They may be employed to project out an eigenvector $\left(\Psi_{n}\right)$ (corresponding to the eigenvalue $\lambda_{n}$ ), given a state vector $\mid \Psi$ ), by operation from the left. They may also be employed to project out desired components of a density operator $\rho$, by the operation $P \rho P$. For our present application, it is of particular interest to note that [2] and [3] imply the representation of any function of an operator $A$ as a polynomial of degree $n,(n+1)$ being the number of distinct eigenvalues of $A$.

In the following, we illustrate the application of these methods to solve spin evolution under various experimental situations, including pulse rotations, spin tickling, spin interferometry, spin locking, and dipolar evolution. In each case, the results are well established theoretically as well as experimentally. These examples permit, however, a rather complete illustration of the projection operator methods for Hamiltonians involving linear as well as bilinear operators in both degenerate and nondegenerate cases. In a subsequent publication, we report on the application of the method to determine the coherence transfer process under isotropic mixing applied to systems of spin- 1 and spin- $\frac{3}{2}$ nuclei.

\section{APPLICATIONS}

Pulse rotation. Consider the effect of a resonant RF pulse with flip angle $\theta$ applied along the $x$ axis in the rotating frame on a system of isolated spins-1. The density matrix of the system following the pulse is given by

$$
\rho_{+}=\exp \left(-i \theta I_{x}\right) \rho_{0} \exp \left(i \theta I_{x}\right)
$$

Wc procecd by first finding a polynomial expression for $\exp \left( \pm i \theta I_{x}\right)$. We note that the eigenvalues of $I_{x}$ are $+1,0$, and -1 , from the fundamental considerations of angular momentum theory. Thus we have

$$
\exp \left( \pm i \theta I_{x}\right)=\exp ( \pm i \theta) P_{1}+P_{0}+\exp (\mp i \theta) P_{-1}
$$


with

$$
\begin{aligned}
P_{1} & =\frac{1}{2} I_{x}\left(I_{x}+1\right)=\frac{1}{2}\left(I_{x}^{2}+I_{x}\right) \\
P_{0} & =-\left(I_{x}-1\right)\left(I_{x}+1\right)=\left(1-I_{x}^{2}\right) \\
P_{-1} & =\frac{1}{2}\left(I_{x}-1\right) I_{x}=\frac{1}{2}\left(I_{x}^{2}-I_{x}\right) .
\end{aligned}
$$

This results finally in

$$
\exp \left( \pm i \theta I_{x}\right)=I_{x}^{2}(\cos \theta-1) \pm i I_{x} \sin \theta+1
$$

With $\rho_{0}=I_{z}$ therefore we have

$$
\begin{aligned}
\rho_{+} & =\left[I_{x}^{2}(\cos \theta-1)-i I_{x} \sin \theta+1\right] I_{z}\left[I_{x}^{2}(\cos \theta-1)+i I_{x} \sin \theta+1\right] \\
& =I_{z} \cos \theta-I_{y} \sin \theta
\end{aligned}
$$

employing the identities $I_{x} I_{z} I_{x}=0$ and $I_{x}^{2} I_{z}+I_{z} I_{x}^{2}=I_{z}$, for $I=1$. For $I=\frac{3}{2}$, on the other hand, we find

$$
\begin{aligned}
\exp \left( \pm i \theta I_{x}\right)= \pm i I_{x}^{3}\left(\frac{1}{3} \sin \frac{3 \theta}{2}\right. & \left.-\sin \frac{\theta}{2}\right)+\frac{1}{2} I_{x}^{2}\left(\cos \frac{3 \theta}{2}-\cos \frac{\theta}{2}\right) \\
\pm & i I_{x}\left(\frac{9}{4} \sin \frac{\theta}{2}-\frac{1}{12} \sin \frac{3 \theta}{2}\right)+\frac{1}{8} 1\left(9 \cos \frac{\theta}{2}-\cos \frac{3 \theta}{2}\right)
\end{aligned}
$$

the eigenvalues of $I_{x}$ being of course $\frac{3}{2}, \frac{1}{2},-\frac{1}{2}$, and $-\frac{3}{2}$ in this case.

Spin tickling. Consider a homonuclear weakly coupled system of two spin- $\frac{1}{2}$ nuclei, I and S. The spin tickling experiment (11) consists in selective irradiation of one of the doublet resonances of, say, spin $S$ while the spectrum is acquired. The Hamiltonian of the system may be written, in the frame rotating with the frequency of irradiation on spin $\mathrm{S}$, as

$$
\mathscr{H}=\Delta I_{z}+\frac{J}{2}\left(1+2 I_{z}\right) S_{z}+\frac{1}{2} \omega_{1}\left(1-2 I_{z}\right) S_{x}
$$

Here, the first term, which commutes with both the others, describes the resonance offset of spin I in this frame, while the second term accounts for the IS weak coupling, as well as the resonance offset of the S-spin irradiation, with $J$ in units of angular frequency. The third term describes the effect of the selective irradiation on one of the S-spin transitions; the Hamiltonian of [10] corresponds in fact to irradiation of the low-frequency component of the S doublet ( $J$ assumed $>0$ ), the RF being along the $x$ axis of the rotating frame. Owing to the commutativity of the first term with the two others, the Hamiltonian of [10] may be rewritten as

$$
\mathscr{H}=\mathscr{H}_{1}+\mathscr{H}_{2}
$$


with

$$
\mathscr{H}_{1}=\Delta I_{z}
$$

and

$$
\mathscr{H}_{2}=\frac{J}{2}\left(1+2 I_{z}\right) S_{z}+\frac{1}{2} \omega_{1}\left(1-2 I_{z}\right) S_{x}
$$

The evolution under $\mathscr{H}$ may be considered as a cascade of independent evolutions under $\mathscr{H}_{1}$ and $\mathscr{H}_{2}$, taken in either order. Here we focus attention on the evolution under $\mathscr{H}_{2}$, which may be rewritten as

$$
\mathscr{H}_{2}=\frac{J}{2}\left[\left(1+2 I_{z}\right) S_{z}+b\left(1-2 I_{z}\right) S_{x}\right]
$$

with $b=\omega_{1} / J$.

To evaluate expressions such as $\exp \left(-i \mathscr{H}_{2} t\right) \rho(0) \exp \left(i \mathscr{H}_{2} t\right)$ we note first that the roots of $\mathscr{H}_{2}$, expressed in units of $J / 2$ are $\pm 1, \pm b$. Thus we have

$$
\begin{aligned}
\exp \left(-i \mathscr{H}_{2} t\right)=\exp (-i J t / 2) P_{1}+\exp (i J t / 2) P_{-1} \\
\\
+\exp (-i J b t / 2) P_{b}+\exp (i J b t / 2) P_{-b}
\end{aligned}
$$

Performing the necessary algebra, we finally find

$$
\begin{aligned}
\exp \left(-i \mathscr{H}_{2} t\right)=\frac{1}{2}( & \left.1+2 I_{z}\right) \cos (J t / 2)+\frac{1}{2}\left(1-2 I_{z}\right) \cos (J b t / 2) \\
& -\frac{i}{2}\left(2 S_{z}+4 I_{z} S_{z}\right) \sin (J t / 2)-\frac{i}{2}\left(2 S_{x}-4 I_{z} S_{x}\right) \sin (J b t / 2)
\end{aligned}
$$

With an initial density operator corresponding to $y$ magnetization of the I spins in the rotating frame, for example, this leads to the state

$$
\begin{array}{r}
\exp \left(-i \mathscr{H}_{2} t\right) I_{y} \exp \left(i \mathscr{H}_{2} t\right)=\frac{1}{2} I_{y}\left[\cos \frac{\left(J+\omega_{1}\right) t}{2}+\cos \frac{\left(J-\omega_{1}\right) t}{2}\right] \\
-I_{x} S_{z}\left[\sin \frac{\left(J+\omega_{1}\right) t}{2}+\sin \frac{\left(J-\omega_{1}\right) t}{2}\right]+I_{x} S_{y}\left[\cos \frac{\left(J-\omega_{1}\right) t}{2}-\cos \frac{\left(J+\omega_{1}\right) t}{2}\right] \\
+I_{x} S_{x}\left[\sin \frac{\left(J+\omega_{1}\right) t}{2}-\sin \frac{\left(J-\omega_{1}\right) t}{2}\right] \cdot
\end{array}
$$

The effect of the I-spin offset may of course simply be cascaded employing $\mathscr{H}_{1}$.

The above expression clearly demonstrates that each of the I-spin transitions is split into a doublet, where the two components have equal intensity and a splitting of $\omega_{1} / 2 \pi \mathrm{Hz}$. Further, the creation of zero- and double-quantum coherences during tickling (12) is also transparent.

Spin interferometry. An elegant, direct demonstration of the spinor properties of spin- $\frac{1}{2}$ particles has been performed by Vaughan and co-workers (13). Their experi- 
ment, carried out on a scalar-coupled heteronuclear spin- $\frac{1}{2}$ pair, may be represented as

$$
\frac{\pi}{2}(I)-\operatorname{Sel} . \mathrm{Irr} .(\tau, S)-(T-\tau)-\pi(I)-T-\Lambda \mathrm{cq} .(I)
$$

This experiment, which they appropriately termed spin interferometry, permits the change in phase of a wavefunction - effected by a pulse rotation-to be observed via a superposition state. Phase differences are observable in superposition states, and by employing a coupled heteronuclear spin as the reference in the supcrposition, the phase change of the other spin is monitored in effect.

Here the selective irradiation is on one of the transitions of the S-spin doublet. The evolution of the I-spin magnetization under the offset term is refocused at the start of acquisition; evolution under heteronuclear coupling during the period $(T-\tau)$ before and after the $\pi$ pulse is also refocused. The observed I-spin signal then is to be computed by taking into account evolution under coupling and selective irradiation during the first $\tau$ period, the effect of the $\pi$ pulse, and evolution under coupling during the last $\tau$ period (just prior to acquisition). In fact, the entire procedure is spin tickling in disguise, and straightforward calculation of the evolution of the first two terms of [15] under the $\pi$ pulse and under coupling for $\tau$ seconds leads to the desired expression for the observable components of the density matrix at the start of acquisition:

$$
\rho(2 T)=I_{y} \cos \left(\omega_{1} \tau / 2\right) .
$$

This clearly demonstrates that rotation of the state of the $S$ spins by a selective pulse with flip angle $\omega_{1} \tau$ has actually resulted in rotation of the wavefunction by $\omega_{1} \tau / 2$, a manifestation of the spinor property of the spin- $\frac{1}{2}$ particlc.

Spin locking. Homonuclear spin-lock experiments have grown in popularity in the last several years and can be described accurately especially at low fields by a model Hamiltonian of the form (14-16)

$$
\mathscr{H}=J \mathbf{I} \cdot \mathbf{S}
$$

It is instructive to compute the evolution of a spin system under this Hamiltonian by employing projection operator methods. For two spins- $\frac{1}{2}$, the Hamiltonian of [17] has the following eigenvalues, in units of $J:+\frac{1}{4}$ (triply degenerate) and $-\frac{3}{4}$. Accordingly, we have

$$
\begin{aligned}
\exp ( \pm i \mathscr{H} l)=\left[\frac{3}{4} \exp ( \pm i J t / 4)+\frac{1}{4} \exp (\mp 3 i J t / 4)\right] 1 \\
+[\exp ( \pm i J t / 4)-\exp (\mp 3 i J t / 4)](\mathbf{I} \cdot \mathbf{S})
\end{aligned}
$$

With an initial density operator $\rho(0)=I_{x}$, we thus find for evolution under spin lock for a period $t$

$$
\begin{aligned}
\rho(t) & =\exp (-i \mathscr{H} t) \rho(0) \exp (i \mathscr{H} t) \\
& =\frac{1}{2} I_{x}(1+\cos J t)+\frac{1}{2} S_{x}(1-\cos J t)+\left(I_{y} S_{z}-I_{z} S_{y}\right) \sin J t
\end{aligned}
$$

employing the identities $I_{l} I_{m}=-I_{m} I_{l}=(i / 2) \epsilon_{l m n} I_{n}+\frac{1}{4} \delta_{l m}$, valid for spin- $\frac{1}{2}$ particles. 
For $I=1=S$, on the other hand, we have the eigenvalues $1,-1$, and -2 in units of $J$, with respective degeneracies of 5,3 , and 1 , leading to

$$
\begin{aligned}
\exp ( \pm i J \mathbf{I} \cdot \mathbf{S} t)= & (\mathbf{I} \cdot \mathbf{S})^{2}\left[\frac{1}{6} \exp ( \pm i J t)-\frac{1}{2} \exp (\mp i J t)+\frac{1}{3} \exp (\mp 2 i J t)\right] \\
& +\frac{1}{2}(\mathbf{I} \cdot \mathbf{S})[\exp ( \pm i J t)-\exp (\mp i J t)] \\
& +\mathbf{1}\left[\exp (\mp i J t)+\frac{1}{3}(\exp ( \pm i J t)-\exp (\mp 2 i J t))\right] .
\end{aligned}
$$

Dipolar evolution. For a homonuclear pair of spin- $\frac{1}{2}$ nuclei, the dipolar Hamiltonian takes the form

$$
\mathscr{H}_{d}=\frac{\gamma^{2} h}{4 \pi r_{12}^{3}}\left(1-3 \cos ^{2} \theta\right)\left(3 I_{z} S_{z}-\mathbf{I} \cdot \mathbf{S}\right)
$$

in the rotating frame, expressed in angular frequency units. The two terms of the last factor commute and their effects may be cascaded. The last term of this Hamiltonian is in fact of the same form as the model Hamiltonian for isotropic mixing under spin locking considered above. The first term, $I_{z} S_{z}$, has the eigenvalues $\pm \frac{1}{4}$, expressed in units of $3 D / 2=3 \gamma^{2} h\left(1-3 \cos ^{2} \theta\right) / 4 \pi r_{12}^{3}$, each of them doubly degenerate. We find

$$
\exp \left( \pm 3 i D I_{z} S_{z} t / 2\right)=\cos (3 D t / 8) 1 \pm 4 i \sin (3 D t / 8) I_{z} S_{z}
$$

which is identical in form to the well-known equation for evolution under weak scalar coupling. This leads to

$$
\begin{aligned}
\exp \left(-i \mathscr{H}_{d} t\right) I_{x} \exp \left(i \mathscr{H}_{d} t\right)=\frac{1}{2} I_{x}[\cos (3 D t / 4)+\cos (D t / 4)] \\
\quad+\frac{1}{2} S_{x}[\cos (3 D t / 4)-\cos (D t / 4)] \\
+I_{y} S_{z}[\sin (3 D t / 4)+\sin (D t / 4)]+S_{y} I_{z}[\sin (3 D t / 4)-\sin (D t / 4)]
\end{aligned}
$$

\section{DISCUSSION}

In the preceding, we have pointed out and demonstrated by way of several illustrations a convenient and straightforward method of dealing with exponential operators, leading to the solution of density operator evolutions. This method of solving cvolutions may be compared with the method of solution employing commutator algebra arising from the Baker-Campbell-Hausdorff formula. In a certain sense, the present method leads to a solution in the frequency domain, while the $\mathrm{BCH}$ method really results in a time-domain solution. The two are, therefore, entirely equivalent as they must be. The $\mathrm{BCH}$ method is probably the method of choice if one is, in particular, interested in short time evolutions, initial rate approximations, etc., and makes the geometry of the evolution process transparent in this sense. On the other hand, the present method is clearly more convenient when one seeks a closed analytical solution of the problem, making as it does the bookkeeping of infinitesimal evolution steps entirely unnecessary: a very important consideration in dealing with complex evolutions, multidimensional spin systems, etc. However, the present method usually carries along a certain amount of irrelevant information: the exponential operator reflects essentially all possible "connectivities" in the system, while the direct 
$\mathrm{BCH}$ method generates only those connectivities that are allowed for a given initial state.

\section{ACKNOWI,FDGMFNTS}

This manuscript was prepared while the author was on a short visit to the Abt. Biomedizinische NMR, Max-Planck-Institut-für biophysikalische Chemie, Göttingen, FRG. It is a pleasure to acknowledge the stimulating and warm ambience I encountered in the group of Dr. J. Frahm.

\section{REFERENCES}

1. U. HAEBERLEN AND J. S. WAUGH, Phys. Rev. 175, 453 (1968).

2. U. HAEBERLEN, "High Resolution NMR in Solids," Academic Press, New York, 1976.

3. J. H. SHIRLEY, Phys. Rev. B. 138, 979 (1965).

4. M. M. MARICQ, Phys. Rev. B. 37,7215(1988).

5. C. N. Banwell and H. Primas, Mol. Phys. 6, 225 (1963).

6. N. Chandrakumar, J. Magn. Reson. 67, 457 (1986).

7. N. Chandrakumar and S. Subramanian, "Modern Techniques in High Resolution FT NMR," Appendix 2, Springer, New York, 1987.

8. J. Katriel, J. Paldus, And R. PaunCz, Int. J. Quantum Chem. 28, 181 ( 1985 ).

9. N. Chandrakumar and S. Subramanian, "Modern Techniques in High Resolution FT NMR," Appendix 1, Springer, New York, 1987.

10. P.-O. Löwdin, Rev. Mod. Phys. 34, 520 (1962).

11. W. A. Anderson and R. Freeman, $J$. Chem. Phys. 37, 85 (1962); R. Freeman and W. A. AnderSON, J. Chem. Phys. 37, 2053 (1962).

12. V. W. Miner and J. H. Prestegard, J. Am. Chem. Soc. 103, 5979 (1981).

13. M. E. Stoll, A. J. Vega, And R. W. Vaughan, Phys. Rev. A 16, 1521 (1977).

14. L. Braunschweiler and R. R. ERnSt, J. Magn. Reson. 53, 521 ( 1983 ).

15. N. Chandrakumar and S. Subramanian, J. Magn. Reson. 62, 346 ( 1985 ).

16. N. Chandrakumak, J. Magn. Reson. 71, 322 (1987). 